\title{
Brouwer degree of polynomial maps
}

\author{
Marek Golasiński • Francisco Gómez Ruiz
}

Dedicated to Hvedri Inassaridze on his 80th birthday

Received: 10 January 2013 / Accepted: 15 July 2013 / Published online: 20 August 2013

(C) The Author(s) 2013. This article is published with open access at Springerlink.com

\begin{abstract}
Let $\mathbb{S}^{n}$ be the $n$-sphere. It is well known that $\pi_{n}\left(\mathbb{S}^{n}\right)=\mathbb{Z}$, and if $n$ is even, $\pi_{2 n-1}\left(\mathbb{S}^{n}\right)=\mathbb{Z} \oplus G$, where $\mathbb{Z}$ is the infinite cyclic group and $G$ a finite abelian group. This allows us to associate an integer to any continuous map $f: \mathbb{S}^{n} \rightarrow \mathbb{S}^{n}$, its Brouwer degree $B(f)$, and to any continuous map $g: \mathbb{S}^{2 n-1} \rightarrow \mathbb{S}^{n}$ for even $n$ its Hopf degree $h(g)$. The purpose of this paper is to define Brouwer and Hopf degree for polynomial maps on spheres over a field of characteristic zero.
\end{abstract}

Keywords Brouwer degree · de Rham cohomology · Hopf degree ·

Polynomial map $\cdot$ Regular (smooth) function

Mathematics Subject Classification (2010) Primary 14P25 - 19A49;

Secondary 55R50

Communicated by Tornike Kadeishvili.

M. Golasiński $(\bowtie)$

Faculty of Mathematics and Computer Science,

University of Warmia and Mazury, Słoneczna 54,

10-710 Olsztyn, Poland

e-mail: marekg@matman.uwm.edu.pl

F. Gómez Ruiz

Departamento de Álgebra, Geometría y Topología, Facultad de Ciencias,

Universidad de Málaga, Campus Universitario de Teatinos,

29071 Málaga, Spain

e-mail: gomez_ruiz@uma.es 


\section{Introduction}

If we take $f: \mathbb{S}^{n} \rightarrow \mathbb{S}^{n}$ smooth and use de Rham differential forms and de Rham cohomology, the induced map $f^{*}$ in $H^{n}\left(\mathbb{S}^{n}\right)$ is multiplication by the Brouwer degree $B(f)$. Also, if we choose the usual $n$-form $\alpha_{n}$, representing the positive orientation on $\mathbb{S}^{n}$ whose integral over the sphere is one, then the Hopf degree $h(g)$ of a smooth $\operatorname{map} g: \mathbb{S}^{2 n-1} \rightarrow \mathbb{S}^{n}$ is given by the formula

$$
\left[\beta_{n-1} g^{*}\left(\alpha_{n}\right)\right]=h(g)\left[\alpha_{2 n-1}\right],
$$

where $d \beta_{n-1}=g^{*}\left(\alpha_{n}\right)$, and [-] means a cohomology class.

Recall that a polynomial map $p=\left(p_{0}, \ldots, p_{n}\right): \mathbb{S}^{m} \rightarrow \mathbb{S}^{n}$ is called a form of degree $k$ if each polynomial $p_{0}, \ldots, p_{n}$ over the reals $\mathbb{R}$ is homogeneous of degree $k$. The result presented in [9, Theorem 1] states: If $n$ is odd then the element of the infinite cyclic group $\pi_{n}\left(\mathbb{S}^{n}\right)$ corresponding to the integer $k$ can be represented by a form of degree $|k|$ mapping $\mathbb{S}^{n}$ to $\mathbb{S}^{n}$. Further, the well-known Hopf maps $\mathbb{S}^{3} \rightarrow \mathbb{S}^{2}$, $\mathbb{S}^{7} \rightarrow \mathbb{S}^{4}$ and $\mathbb{S}^{15} \rightarrow \mathbb{S}^{8}$ are given by forms of degree 2 . In particular, elements of the infinite cyclic group $\pi_{3}\left(\mathbb{S}^{2}\right)$ are represented by forms.

If $\mathbb{C}$ is the field of complex numbers, then the complex $n$-sphere

$$
\mathbb{S}^{n}(\mathbb{C})=\left\{\left(z_{0}, \ldots, z_{n}\right) \in \mathbb{C}^{n+1} ; z_{0}^{2}+\cdots+z_{n}^{2}=1\right\}
$$

has the homotopy type of $\mathbb{S}^{n}$ as diffeomorphic to the tangent bundle $T \mathbb{S}^{n}$ and by [10, Theorem 2]: Every element in $\pi_{n}\left(\mathbb{S}^{n}\right)$ can be represented by a complex polynomial map of $\mathbb{S}^{n}(\mathbb{C})$ for all positive integers $n$.

This inspires us to present the notions above in a more algebraic setting. We replace the reals $\mathbb{R}$ by a field $\mathbb{F}$ of characteristic zero; the algebra of smooth functions $C^{\infty}\left(\mathbb{B}^{n}\right)$ on the $n$-ball $\mathbb{B}^{n}$, by a commutative $\mathbb{F}$-algebra $\mathcal{A}$ such that $\mathbb{F}\left[X_{1}, \ldots, X_{n}\right] \subseteq \mathcal{A}$; the $C^{\infty}\left(\mathbb{B}^{n}\right)$-free module $\Omega^{1}\left(\mathbb{B}^{n}\right)$ of smooth 1 -forms on $\mathbb{B}^{n}$, by an $\mathcal{A}$-free module $\Omega^{1}(\mathcal{A})$ together with an $\mathbb{F}$-linear map $d: \mathcal{A} \rightarrow \Omega^{1}(\mathcal{A})$ such that $d(a b)=a d b+b d a$ for all $a, b \in \mathcal{A}$ and with $d X_{1}, \ldots, d X_{n}$ being a basis. Finally, the algebra of real smooth functions $C^{\infty}\left(\mathbb{S}^{n}\right)$ is replaced by $\mathcal{B}=\mathcal{A}\left[X_{0}, \ldots, X_{n}\right] /\left(\sum_{j=0}^{n} X_{j}^{2}-1\right)$.

In the second section, we give several examples and show in particular that

$$
C^{\infty}\left(\mathbb{B}^{n}\right)\left[X_{0}\right] /\left(\sum_{j=0}^{n} X_{j}^{2}-1\right) \stackrel{\cong}{\longrightarrow} C^{\infty}\left(\mathbb{S}^{n}\right)
$$

is an isomorphism.

We associate the "de Rham cohomology" to $\mathcal{B}$ and give sufficient conditions so that $H^{k}(\mathcal{B}) \neq 0$ for $k=0, n$ and both $H^{0}(\mathcal{B})$ and $H^{n}(\mathcal{B})$ are isomorphic to $\mathbb{F}$.

Then, we define the Brouwer degree $B(p)$ of a polynomial map $p: \mathbb{S}^{n}(\mathbb{F}) \rightarrow \mathbb{S}^{n}(\mathbb{F})$, and prove that $B(p)$ is an integer.

The third section imitates the classical case to define a Hopf degree $h(q)$ for any polynomial map $q: \mathbb{S}^{2 n-1}(\mathbb{F}) \rightarrow \mathbb{S}^{n}(\mathbb{F})$ for even $n$ and then we show that $h(q)$ is an integer. 


\section{Brouwer degree}

Let $\mathbb{F}$ be a field of characteristic zero and $\mathcal{A}$ a commutative $\mathbb{F}$-algebra such that the polynomial ring $\mathbb{F}\left[X_{1}, \ldots, X_{n}\right] \subseteq \mathcal{A}$. Assume that there is also given an $\mathcal{A}$-free module $\Omega^{1}(\mathcal{A})$, together with an $\mathbb{F}$-linear map $d: \mathcal{A} \rightarrow \Omega^{1}(\mathcal{A})$ such that $d(a b)=$ $a d b+b d a$ for $a, b \in \mathcal{A}$ and with $d X_{1}, \ldots, d X_{n}$ being a basis of $\Omega^{1}(\mathcal{A})$.

Given $a \in \mathcal{A}$, define $\frac{\partial a}{\partial X_{i}}$ for $i=1, \ldots, n$ by the formula $d a=\sum_{j=1}^{n} \frac{\partial a}{\partial X_{j}} d X_{j}$. We further suppose that $\frac{\partial}{\partial X_{i}} \frac{\partial a}{\partial X_{j}}=\frac{\partial}{\partial X_{j}} \frac{\partial a}{\partial X_{i}}$ for $1 \leq i<j \leq n$. This implies, in particular, that $d$ can be extended to a differential on the exterior powers $\Omega^{k}(\mathcal{A})$ of $\Omega^{1}(\mathcal{A})$ for $k \geq 1$.

Example 2.1 1. For $\mathcal{A}=\mathcal{A}_{n}=\mathbb{F}\left[X_{1}, \ldots, X_{n}\right]$, take $\Omega^{1}\left(\mathcal{A}_{n}\right)$ as the $\mathcal{A}_{n}$-module of Kähler differentials.

2. For the field of reals $\mathbb{R}$, take $\mathcal{A}=C^{\infty}\left(\mathbb{R}^{n}\right)$, the ring of smooth functions on $\mathbb{R}^{n}$ or $\mathcal{A}=C^{\infty}\left(\mathbb{B}^{n}\right)$, the ring of smooth functions on the $n$-ball $\mathbb{B}^{n}$ and $\Omega^{1}(\mathcal{A})$ the module of smooth 1 -forms on $\mathbb{R}^{n}$ or $\mathbb{B}^{n}$ respectively and $d$ the exterior derivative.

3. The ring $\mathcal{A}$ of regular functions on $\mathbb{F}^{n}$ and $\Omega^{1}(\mathcal{A})$ the $\mathcal{A}$-module of Kähler differentials.

Then, consider the $\mathbb{F}$-algebra $\mathcal{B}=\mathcal{A}\left[X_{0}\right] /\left(\sum_{j=0}^{n} X_{j}^{2}-1\right)$. Notice that there is an inclusion $\mathcal{A} \subseteq \mathcal{B}$ and elements of $\mathcal{B}$ are uniquely of the form $\alpha+X_{0} \beta$ with $\alpha, \beta \in \mathcal{A}$ and

$$
\left(\alpha+X_{0} \beta\right)\left(\alpha^{\prime}+X_{0} \beta^{\prime}\right)=\alpha \alpha^{\prime}+\beta \beta^{\prime}\left(1-\sum_{j=1}^{n} X_{j}^{2}\right)+X_{0}\left(\alpha \beta^{\prime}+\beta \alpha^{\prime}\right)
$$

in the ring $\mathcal{B}$.

For $\mathcal{A}=\mathcal{A}_{n}$, the $\operatorname{ring} \mathcal{B}=\mathcal{A}\left[X_{0}\right] /\left(\sum_{j=0}^{n} X_{j}^{2}-1\right)$ coincides with polynomial functions

$$
\mathbb{F}\left[X_{0}, \ldots, X_{n}\right] /\left(\sum_{j=0}^{n} X_{j}^{2}-1\right)
$$

on the $n$-sphere

$$
\mathbb{S}^{n}(\mathbb{F})=\left\{\left(x_{0}, \ldots, x_{n}\right) \in \mathbb{F}^{n+1} ; x_{0}^{2}+\cdots+x_{n}^{2}=1\right\}
$$

over $\mathbb{F}$. However, for the ring $\mathcal{A}$ of regular functions on $\mathbb{F}^{n}$, the $\operatorname{ring} \mathcal{B}=$ $\mathcal{A}\left[X_{0}\right] /\left(\sum_{j=0}^{n} X_{j}^{2}-1\right)$ coincides with regular functions on $\mathbb{S}^{n}(\mathbb{F})$.

Now, write $C^{\infty}\left(\mathbb{S}^{n}\right)$ for the ring of smooth functions on the $n$-sphere $\mathbb{S}^{n}$. Because of the inclusions $C^{\infty}\left(\mathbb{R}^{n}\right)\left[X_{0}\right] \subseteq C^{\infty}\left(\mathbb{R}^{n+1}\right)$ and $C^{\infty}\left(\mathbb{B}^{n}\right)\left[X_{0}\right] \subseteq C^{\infty}\left(\mathbb{B}^{n+1}\right)$, the restriction maps

$$
C^{\infty}\left(\mathbb{R}^{n}\right)\left[X_{0}\right] \rightarrow C^{\infty}\left(\mathbb{S}^{n}\right), \quad \text { and } \quad C^{\infty}\left(\mathbb{B}^{n}\right)\left[X_{0}\right] \rightarrow C^{\infty}\left(\mathbb{S}^{n}\right)
$$


lead to monomorphisms

$$
C^{\infty}\left(\mathbb{R}^{n}\right)\left[X_{0}\right] / I \longrightarrow C^{\infty}\left(\mathbb{S}^{n}\right) \text { and } C^{\infty}\left(\mathbb{B}^{n}\right)\left[X_{0}\right] /\left(\sum_{j=0}^{n} X_{j}^{2}-1\right) \longrightarrow C^{\infty}\left(\mathbb{S}^{n}\right)
$$

with a factorisation

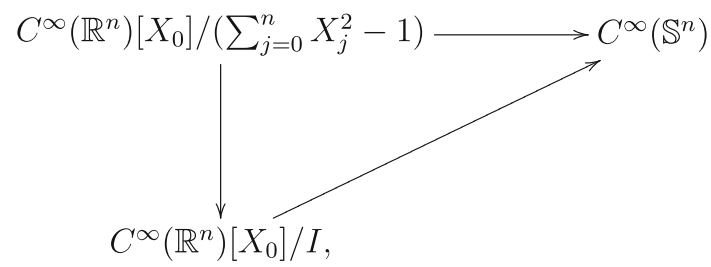

where the ideal $I \subseteq C^{\infty}\left(\mathbb{R}^{n}\right)\left[X_{0}\right]$ is generated by functions in $C^{\infty}\left(\mathbb{R}^{n}\right)$ vanishing on $\mathbb{B}^{n}$.

To show the surjectivity of $C^{\infty}\left(\mathbb{B}^{n}\right)\left[X_{0}\right] /\left(\sum_{j=1}^{n} X_{j}^{2}-1\right) \longrightarrow C^{\infty}\left(\mathbb{S}^{n}\right)$, we recall:

Lemma 2.2 ([2, Chapter VI, Theorem 5.1]) Let $f: \mathbb{R}^{+} \times \mathbb{R}^{n} \rightarrow \mathbb{R}$. If the function

$$
\tilde{f}: \mathbb{R}^{n+1} \longrightarrow \mathbb{R}
$$

given by $\tilde{f}\left(x_{0}, x_{1}, \ldots, x_{n}\right)=f\left(x_{0}^{2}, x_{1}, \ldots, x_{n}\right)$ for $\left(x_{0}, x_{1}, \ldots, x_{n}\right) \in \mathbb{R}^{n+1}$ is smooth then $f$ is smooth as well.

Now, we are ready to show:

Proposition 2.3 If $f: \mathbb{S}^{n} \rightarrow \mathbb{R}$ is a smooth function, then there exist unique real smooth functions $f_{0}, f_{1}: \mathbb{B}^{n} \rightarrow \mathbb{R}$ such that

$$
f\left(x_{0}, \ldots, x_{n}\right)=f_{0}\left(x_{1}, \ldots, x_{n}\right)+x_{0} f_{1}\left(x_{1}, \ldots, x_{n}\right)
$$

for $\left(x_{0}, \ldots, x_{n}\right) \in \mathbb{S}^{n}$.

Proof Notice that $\left( \pm \sqrt{1-\sum_{j=1}^{n} x_{j}^{2}}, x_{1}, \ldots, x_{n}\right) \in \mathbb{S}^{n}$ for any $\left(x_{1}, \ldots, x_{n}\right) \in \mathbb{B}^{n}$. Then, the uniqueness of smooth functions $f_{0}, f_{1}: \mathbb{B}^{n} \rightarrow \mathbb{R}$ is clear because

$$
f\left(\sqrt{1-\sum_{j=1}^{n} x_{j}^{2}}, x_{1}, \ldots, x_{n}\right)=f_{0}\left(x_{1}, \ldots, x_{n}\right)+\sqrt{1-\sum_{j=1}^{n} x_{j}^{2}} f_{1}\left(x_{1}, \ldots, x_{n}\right)
$$

and

$$
f\left(-\sqrt{1-\sum_{j=1}^{n} x_{j}^{2}}, x_{1}, \ldots, x_{n}\right)=f_{0}\left(x_{1}, \ldots, x_{n}\right)-\sqrt{1-\sum_{j=1}^{n} x_{j}^{2}} f_{1}\left(x_{1}, \ldots, x_{n}\right)
$$

for all $\left(x_{1}, \ldots, x_{n}\right) \in \mathbb{B}^{n}$. 
To show an existence, given a smooth $f: \mathbb{S}^{n} \rightarrow \mathbb{R}$, we define:

$$
\begin{aligned}
f_{0}\left(x_{1}, \ldots, x_{n}\right)= & \frac{1}{2}\left(f\left(\sqrt{1-\sum_{j=1}^{n} x_{j}^{2}}, x_{1}, \ldots, x_{n}\right)\right. \\
& \left.+f\left(-\sqrt{1-\sum_{j=1}^{n} x_{j}^{2}}, x_{1}, \ldots, x_{n}\right)\right)
\end{aligned}
$$

for any $\left(x_{1}, \ldots, x_{n}\right) \in \mathbb{B}^{n}$ and

$$
\begin{aligned}
f_{1}\left(x_{1}, \ldots, x_{n}\right)= & \frac{1}{2 \sqrt{1-\sum_{j=1}^{n} x_{j}^{2}}}\left(f\left(\sqrt{1-\sum_{j=1}^{n} x_{j}^{2}}, x_{1}, \ldots, x_{n}\right)\right. \\
& \left.-f\left(-\sqrt{1-\sum_{j=1}^{n} x_{j}^{2}}, x_{1}, \ldots, x_{n}\right)\right)
\end{aligned}
$$

for any $\left(x_{1}, \ldots, x_{n}\right) \in \dot{\mathbb{B}}^{n}$, the interior of $\mathbb{B}^{n}$.

Observe that $f_{0}\left(x_{1}, \ldots, x_{n}\right)=f\left(0, x_{1}, \ldots, x_{n}\right)$ whenever $\sum_{j=1}^{n} x_{j}^{2}=1$ and it is obvious that $f_{0}$ and $f_{1}$ are smooth in the interior of $\mathbb{B}^{n}$. It remains to show that $f_{1}$ extend continuously to the frontier of $\mathbb{B}^{n}$ and both $f_{0}$ and $f_{1}$ are also smooth on this frontier.

Now, in view of Tubular Neighborhood Theorem [7, Chapter 4, Theorem 5.1], $f: \mathbb{S}^{n} \rightarrow \mathbb{R}$ extends to a smooth function $\tilde{f}: \mathbb{R}^{n+1} \rightarrow \mathbb{R}$. Next, denote by $\mathbb{R}^{+}$the set of nonnegative reals and define $g: \mathbb{R}^{+} \times \mathbb{R}^{n} \rightarrow \mathbb{R}$ by

$$
g\left(x_{0}, \ldots, x_{n}\right)=\frac{1}{2}\left(\tilde{f}\left(\sqrt{x_{0}}, x_{1}, \ldots, x_{n}\right)+\tilde{f}\left(-\sqrt{x_{0}}, x_{1}, \ldots, x_{n}\right)\right)
$$

for $\left(x_{0}, \ldots, x_{n}\right) \in \mathbb{R}^{+} \times \mathbb{R}^{n}$ and $\tilde{g}: \mathbb{R}^{n+1} \rightarrow \mathbb{R}$ by

$$
\tilde{g}\left(x_{0}, \ldots, x_{n}\right)=\frac{1}{2}\left(\tilde{f}\left(x_{0}, x_{1}, \ldots, x_{n}\right)+\tilde{f}\left(-x_{0}, x_{1}, \ldots, x_{n}\right)\right)=g\left(x_{0}^{2}, x_{1}, \ldots, x_{n}\right)
$$

for $\left(x_{0}, \ldots, x_{n}\right) \in \mathbb{R}^{n+1}$.

Because $\tilde{g}$ is smooth, Lemma 2.2 yields that $g$ is smooth as well. Then,

$$
f_{0}\left(x_{1}, \ldots, x_{n}\right)=g\left(1-\sum_{j=1}^{n} x_{j}^{2}, x_{1}, \ldots, x_{n}\right)
$$

for $\left(x_{1}, \ldots, x_{n}\right) \in \mathbb{R}^{n}$ yields that $f_{0}: \mathbb{B}^{n} \rightarrow \mathbb{R}$ is also a smooth function. 
Next, define a smooth function $\tilde{h}: \mathbb{R}^{n+1} \rightarrow \mathbb{R}$ by

$$
\tilde{h}\left(x_{0}, \ldots, x_{n}\right)=\frac{1}{2}\left(\tilde{f}\left(x_{0}, x_{1}, \ldots, x_{n}\right)-\tilde{f}\left(-x_{0}, x_{1}, \ldots, x_{n}\right)\right)
$$

for $\left(x_{0}, \ldots, x_{n}\right) \in \mathbb{R}^{n+1}$.

Notice that

$$
\tilde{f}=\tilde{g}+\tilde{h}
$$

and

$$
\tilde{h}\left(x_{0}, \ldots, x_{n}\right)=x_{0} f_{1}\left(x_{1}, \ldots, x_{n}\right)
$$

provided $\sum_{j=0}^{n} x_{j}=1$ and $x_{0} \neq 0$.

Furthermore, we have

$$
\frac{\partial \tilde{h}}{\partial x_{0}}\left(0, x_{1}, \ldots, x_{n}\right)=\lim _{x_{0} \rightarrow 0} \frac{\tilde{h}\left(x_{0}, \ldots, x_{n}\right)}{x_{0}} .
$$

It is clear that the function $\bar{h}: \mathbb{R}^{n+1} \rightarrow \mathbb{R}$ given by

$$
\bar{h}\left(x_{0}, \ldots, x_{n}\right)= \begin{cases}\frac{\tilde{h}\left(x_{0}, \ldots, x_{n}\right)}{x_{0}}, & \text { if } x_{0} \neq 0 \\ \frac{\partial \tilde{h}}{\partial x_{0}}\left(0, x_{1}, \ldots, x_{n}\right) & \text { if } x_{0}=0\end{cases}
$$

for $\left(x_{0}, \ldots, x_{n}\right) \in \mathbb{R}^{n+1}$ is smooth and

$$
\tilde{h}\left(x_{0}, \ldots, x_{n}\right)=x_{0} \bar{h}\left(x_{0}, \ldots, x_{n}\right)
$$

for all $\left(x_{0}, \ldots, x_{n}\right) \in \mathbb{R}^{n+1}$.

Observe that $\bar{h}\left(x_{0}, \ldots, x_{n}\right)=f_{1}\left(x_{1}, \ldots, x_{n}\right)$ provided $\sum_{j=0}^{n} x_{j}^{2}=1$ and $x_{0} \neq 0$. Therefore $f_{1}$ extends continuously to the frontier of $\mathbb{B}^{n}$ by defining $f_{1}\left(x_{1}, \ldots, x_{n}\right)=$ $\bar{h}\left(0, x_{1}, \ldots, x_{n}\right)$ whenever $\sum_{j=1}^{n} x_{j}^{2}=1$.

At the end, defining $h: \mathbb{R}^{+} \times \mathbb{R}^{n} \rightarrow \mathbb{R}$ by

$$
h\left(x_{0}, \ldots, x_{n}\right)=\bar{h}\left(\sqrt{x_{0}}, x_{1}, \ldots, x_{n}\right)=\bar{h}\left(-\sqrt{x_{0}}, x_{1}, \ldots, x_{n}\right)
$$

for $\left(x_{0}, \ldots, x_{n}\right) \in \mathbb{R}^{+} \times \mathbb{R}^{n}$, we have

$$
h\left(x_{0}^{2}, x_{1}, \ldots, x_{n}\right)=\bar{h}\left(x_{0}, x_{1}, \ldots, x_{n}\right)
$$

for all $\left(x_{0}, x_{1}, \ldots, x_{n}\right) \in \mathbb{R}^{n+1}$. Then, Lemma 2.2 implies that $h$ is smooth. Because $f_{1}\left(x_{1}, \ldots, x_{n}\right)=h\left(1-\sum_{j=1}^{n} x_{j}^{2}, x_{1}, \ldots, x_{n}\right)$ for $\left(x_{1}, \ldots, x_{n}\right) \in \mathbb{B}^{n}$, we deduce that the function $f_{1}$ is smooth and the proof is complete. 
Because $\mathbb{B}^{n}$ is compact, by means of the smooth Tietze-Urysohn extension theorem, the restriction map $C^{\infty}\left(\mathbb{R}^{n}\right)\left[X_{0}\right] \longrightarrow C^{\infty}\left(\mathbb{B}^{n}\right)\left[X_{0}\right]$ is an epimorphism; we conclude:

Corollary 2.4 The restriction maps $C^{\infty}\left(\mathbb{B}^{n}\right)\left[X_{0}\right] \rightarrow C^{\infty}\left(\mathbb{S}^{n}\right)$ and $C^{\infty}\left(\mathbb{R}^{n}\right)\left[X_{0}\right] \rightarrow$ $C^{\infty}\left(\mathbb{S}^{n}\right)$ lead to isomorphisms

$$
C^{\infty}\left(\mathbb{B}^{n}\right)\left[X_{0}\right] /\left(\sum_{j=0}^{n} X_{j}^{2}-1\right) \stackrel{\cong}{\longrightarrow} C^{\infty}\left(\mathbb{S}^{n}\right) \text { and } C^{\infty}\left(\mathbb{R}^{n}\right)\left[X_{0}\right] / I \stackrel{\cong}{\longrightarrow} C^{\infty}\left(\mathbb{S}^{n}\right),
$$

and an epimorphism

$$
C^{\infty}\left(\mathbb{R}^{n}\right)\left[X_{0}\right] /\left(\sum_{j=0}^{n} X_{j}^{2}-1\right) \longrightarrow C^{\infty}\left(\mathbb{S}^{n}\right)
$$

with a factorisation

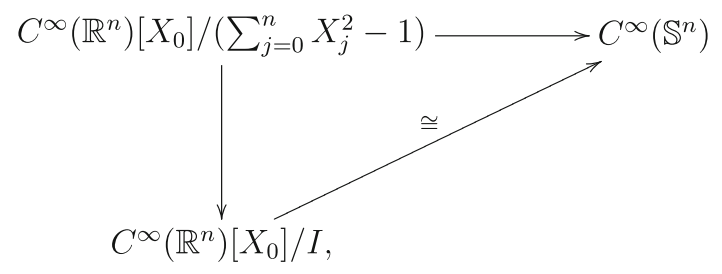

where the ideal $I \subseteq C^{\infty}\left(\mathbb{R}^{n}\right)\left[X_{0}\right]$ is generated by functions in $C^{\infty}\left(\mathbb{R}^{n}\right)$ vanishing on $\mathbb{B}^{n}$.

Let now $\Omega^{1}(\mathcal{B})=\Omega^{1}(\mathcal{A}) \otimes_{\mathcal{A}} \mathcal{B}$ be the $\mathcal{B}$-module which, as an $\mathcal{A}$-module, is free with the basis

$$
d X_{0}, \ldots, d X_{n}, X_{0} d X_{1}, \ldots, X_{0} d X_{n}
$$

and the obvious $\mathcal{B}$-module structure, using the relations

$$
\sum_{j=0}^{n} X_{j}^{2}=1 \text { and } \sum_{j=0}^{n} X_{j} d X_{j}=0
$$

Notice that $\Omega^{1}(\mathcal{B})=\Omega(\mathcal{B})$, the $\mathcal{B}$-module of Kähler differentials for $\mathcal{A}=\mathcal{A}_{n}=$ $\mathbb{F}\left[X_{1}, \ldots, X_{n}\right]$ and $\Omega^{1}(\mathcal{B})$ is the $\mathcal{B}$-module of real smooth 1 -forms on $\mathbb{S}^{n}$ for $\mathcal{A}=$ $C^{\infty}\left(\mathbb{B}^{n}\right)$.

We extend $d$ in the natural way to $d: \mathcal{B} \rightarrow \Omega^{1}(\mathcal{B})$ and then to the exterior powers $\Omega^{k}(\mathcal{B})$ of $\Omega^{1}(\mathcal{B})$. Then, $\Omega^{n+1}(\mathcal{B})=0$ as the $\mathcal{B}$-module generated by $d X_{0} \cdots d X_{n}=0$ and certainly $\Omega^{k}(\mathcal{B})=0$ for $k>n+1$. In fact,

$X_{j} d X_{0} \cdots d X_{n}=d X_{0} \cdots\left(-X_{0} d X_{0}-\cdots-{\widehat{X}, X_{j}}_{j}-\cdots-X_{n} d X_{n}\right) \cdots d X_{n}=0$

and so $d X_{0} \cdots d X_{n}=\left(\sum_{j=0}^{n} X_{j}^{2}\right) d X_{0} \cdots d X_{n}=0$. 
Lemma 2.5 The $\mathcal{B}$-module $\Omega^{n}(\mathcal{B})$ is free and generated by

$$
\omega_{n}=\sum_{i=0}^{n}(-1)^{i} X_{i} d X_{0} \cdots \widehat{d X_{i}} \cdots d X_{n}
$$

Proof Notice that $X_{j} \omega_{n}=(-1)^{j} d X_{0} \cdots \widehat{d X_{j}} \cdots d X_{n}$ for $j=0, \ldots, n$. Given $\Phi \in$ $\Omega^{n}(\mathcal{B})$, we have

$$
\Phi=\sum_{i=0}^{n} \alpha_{i} d X_{0} \cdots \widehat{d X_{i}} \cdots d X_{n}
$$

for some $\alpha_{i} \in B$ with $i=1, \ldots, n$. Thus,

$$
\Phi=\left(\sum_{i=0}^{n}(-1)^{i} \alpha_{i} X_{i}\right) \omega_{n}
$$

Finally, suppose $\left(a+b X_{0}\right) \omega_{n}=0$ with $a, b \in A$. Then,

$0=b d X_{1} \cdots d X_{n}+X_{0} a d X_{1} \cdots d X_{n}+\left(d X_{0}\right) a \sum_{j=1}^{n}(-1)^{j} X_{j} d X_{1} \cdots \widehat{d X_{j}} \cdots d X_{n}$

and so $a=b=0$.

Write $H^{k}(\mathcal{A})$, (resp. $H^{k}(\mathcal{B})$ ) for the cohomology of the complexes $\Omega^{k}(\mathcal{A})$ (resp. $\left.\Omega^{k}(\mathcal{B})\right)$ defined above. Then, we have the following:

Theorem 2.6 1. $H^{k}(\mathcal{B})=0$ for all $k>n$;

2. $H^{0}(\mathcal{B})=\mathbb{F}$ provided that $A \cap \overline{\mathbb{F}}=\mathbb{F}$, where $\overline{\mathbb{F}}$ is the algebraic closure of $\mathbb{F}$;

3. $H^{k}(\mathcal{B})=0$ for $0<k<n$ provided that $H^{k}(\mathcal{A})=0$ for all $k \geq 1$;

4. $H^{n}(\mathcal{B}) \cong \mathbb{F}$ provided there is an $\mathbb{F}$-linear map $\varphi: \mathcal{A} \rightarrow \mathbb{F}$ such that $\varphi(1)=1$ and the kernel of $\varphi$ is generated over $\mathbb{F}$ by the elements of the form $X_{i} a-(1-$ $\left.\sum_{j=1}^{n} X_{j}^{2}\right) \frac{\partial a}{\partial X_{i}}$ for $i=1, \ldots, n$ and $a \in A$.

Proof 1. This is clear because $\Omega^{k}(\mathcal{B})=0$ for $k>n$.

2. Let $\alpha$ and $\beta$ elements of $\mathcal{A}$ such that $d\left(\alpha+X_{0} \beta\right)=0$. Therefore, $d \alpha=0, d \beta=0$ and $\beta=0$ and, in view of [5, Proposition 7], there exist such $\lambda_{0}, \ldots, \lambda_{m} \in \mathbb{F}$ with $\lambda_{m} \neq 0, m \geq 1$, that $\lambda_{0}+\lambda_{1} \alpha+\cdots+\lambda_{m} \alpha^{m}=0$. Whence, $\alpha \in \overline{\mathbb{F}} \cap \mathcal{A}=\mathbb{F}$.

3. As a consequence of the relations $\sum_{j=0}^{n} X_{j}^{2}=1$ and $\sum_{j=0}^{n} X_{j} d X_{j}=0$, we see that for $0 \leq k \leq n$, any element of $\Phi \in \Omega^{k}(\mathcal{B})$ can be written uniquely as a sum

$$
\Phi=\Phi_{1}+X_{0} \Phi_{2}+d X_{0} \Phi_{3}
$$

with $\Phi_{1}, \Phi_{2} \in \Omega^{k}(A) \subseteq \Omega^{k}(\mathcal{B})$ and $\Phi_{3} \in \Omega^{k-1}(\mathcal{A}) \subseteq \Omega^{k-1}(\mathcal{B})$. 
Suppose now that $d \Phi=0$. Then,

$$
0=d \Phi_{1}+X_{0} d \Phi_{2}+d X_{0}\left(\Phi_{2}-d \Phi_{3}\right)
$$

It is clear that the above relation says nothing if $k=n$. But, for $0 \leq k<n$ we have $d \Phi_{1}=0, d \Phi_{2}=0$ and $\Phi_{2}=d \Phi_{3}$. Therefore,

$$
\Phi=\Phi_{1}+d\left(X_{0} \Phi_{3}\right) .
$$

Because, by hypothesis that $H_{d R}^{k}(\mathcal{A})=0$ for $k>0$, there exists $\Psi_{1} \in \Omega^{k-1}(\mathcal{A}) \subseteq$ $\Omega^{k-1}(\mathcal{B})$ such that $\Phi_{1}=d \Psi_{1}$, so we get

$$
\Phi=d\left(\Psi_{1}+X_{0} \Phi_{3}\right)
$$

4. First, observe that any element of $\Omega^{n-1}(\mathcal{B})$ is a sum of elements of the form:

(a) $a d X_{1} \cdots \widehat{d X_{i}} \cdots d X_{n}$, for $a \in \mathcal{A}$ and $i=1, \ldots, n$;

(b) $a X_{0} d X_{1} \cdots \widehat{d X_{i}} \cdots d X_{n}$, for $a \in \mathcal{A}$ and $i=1, \ldots, n$;

(c) $a d X_{0} d X_{1} \cdots \widehat{d X_{i}} \cdots \widehat{d X_{j}} \cdots d X_{n}$, for $a \in \mathcal{A}$ and $1 \leq i<j \leq n$.

Next, in view of Lemma 2.5, the map $\tilde{\varphi}: \Omega^{n}(\mathcal{B})=\mathcal{B} \omega_{n} \rightarrow \mathbb{F}$, given by $\tilde{\varphi}((a+$ $\left.\left.X_{0} b\right) \omega_{n}\right)=\varphi(a)$, for $a, b \in \mathcal{A}$ is a well-defined $\mathbb{F}$-linear extension of $\varphi: \mathcal{A} \rightarrow \mathbb{F}$.

We show that $\tilde{\varphi} \circ d=0$ :

(a) $\tilde{\varphi}\left(d\left(a d X_{1} \cdots \widehat{d X_{i}} \cdots d X_{n}\right)\right)=\varphi(0)=0$;

(b) $d\left(a X_{0} d X_{1} \cdots \widehat{d X_{i}} \cdots d X_{n}\right)=(-1)^{i-1} X_{0} \frac{\partial a}{\partial X_{i}} d X_{1} \cdots d X_{n}+a d X_{0} \cdots \widehat{d X_{i}} \cdots$ $d X_{n}=(-1)^{i} X_{i} a \omega_{n}$ and so $\tilde{\varphi}\left(d\left(a X_{0} d X_{1} \cdots \widehat{d X_{i}} \cdots d X_{n}\right)\right)=0$

(c) $d\left(a d X_{0} d X_{1} \cdots \widehat{d X_{i}} \cdots \widehat{d X_{j}} \cdots d X_{n}\right)=\left(\frac{\partial a}{\partial X_{i}} d X_{i}+\frac{\partial a}{\partial X_{j}} d X_{j}\right) d X_{0} d X_{1} \cdots \widehat{d X_{i}}$ $\cdots \widehat{d X_{j}} \cdots d X_{n}=(-1)^{i-1} \frac{\partial a}{\partial X_{i}} d X_{0} \cdots \widehat{d X_{j}} \cdots d X_{n}+(-1)^{j} \frac{\partial a}{\partial X_{j}} d X_{0} \cdots \widehat{d X_{i}} \cdots$ $d X_{n}=(-1)^{i+j}\left(-X_{j} \frac{\partial a}{\partial X_{i}}+X_{i} \frac{\partial a}{\partial X_{j}}\right) \omega_{n}$.

But, $X_{i} \frac{\partial a}{\partial X_{j}}-\left(1-\sum_{k=1}^{n} X_{k}^{2}\right) \frac{\partial}{\partial X_{i}}\left(\frac{\partial a}{\partial X_{j}}\right), X_{j} \frac{\partial a}{\partial X_{i}}-\left(1-\sum_{k=1}^{n} X_{k}^{2}\right) \frac{\partial}{\partial X_{j}}\left(\frac{\partial a}{\partial X_{i}}\right) \in \operatorname{ker} \varphi$.

Hence, $\tilde{\varphi}\left(d\left(a d X_{0} d X_{1} \cdots \widehat{d X_{i}} \cdots \widehat{d X_{j}} \cdots d X_{n}\right)\right)=\varphi\left(X_{i} \frac{\partial a}{\partial X_{j}}-X_{j} \frac{\partial a}{\partial X_{i}}\right)=\varphi((1-$ $\left.\left.\sum_{k=1}^{n} X_{k}^{2}\right)\left(\frac{\partial}{\partial X_{i}}\left(\frac{\partial a}{\partial X_{j}}\right)-\frac{\partial}{\partial X_{j}}\left(\frac{\partial a}{\partial X_{i}}\right)\right)\right)=0$ and the proof is complete.

Remark 2.7 In Example 1.1(1), $\varphi$ is the map appearing in [8] and in Example 1.1(2), $\varphi(a)=\int_{\mathbb{S}^{n}} a \omega_{n}$, for $a \in \mathcal{A}$, where $\omega_{n}$ is the volume $n$-form. Also, in both cases, it is well known that $H^{k}(\mathcal{A})=0$ for any $k>0$ (see [1] for the case of polynomial algebra), and the elements of the form $X_{i} a-\left(1-\sum_{j=1}^{n} X_{j}^{2}\right) \frac{\partial a}{\partial X_{i}}$ generate the kernel of $\varphi$. Instead in Example 1.1(3) is not true that $H^{k}(\mathcal{A})=0$ for $k>0$. For instance for $\mathbb{F}$ not containing the square root of $-1, d\left(\frac{d X}{X^{2}+1}\right)=0$, but $\frac{d X}{X^{2}+1}$ is not $d f$ for any $f \in \mathcal{A}$.

Given a polynomial map $p=\left(p_{0}, \ldots, p_{n}\right): \mathbb{S}^{n}(\mathbb{F}) \rightarrow \mathbb{S}^{n}(\mathbb{F})$, we have $p^{*}\left(\left[\omega_{n}\right]\right)=$ $B(p)\left[\omega_{n}\right]$ with $B(p) \in \mathbb{F}$. We say that $B(p)$ is the Brouwer degree of $p$. 
Observe that if $p_{j}=\sum a_{\alpha_{0}, \ldots, \alpha_{n}}^{j} X_{0}^{\alpha_{0}} \cdots X_{n}^{\alpha_{n}}$ with $j=0, \ldots, n$, where $a_{\left(\alpha_{0}, \ldots, \alpha_{n}\right)}^{j} \in$ $\mathbb{F}$ and $\alpha=\left(\alpha_{0}, \ldots, \alpha_{n}\right) \in \mathbb{N}^{n+1}$ for the set $\mathbb{N}$ of natural numbers then

$$
B(p) \in \mathbb{Q}\left[a_{\alpha}^{j}\right]_{0 \leq j \leq n ; \alpha \in \mathbb{N}^{n+1}},
$$

i.e., the Brouwer degree $B(p)$ is a polynomial over rationals $\mathbb{Q}$ on the coefficients of the $p_{j}$ for $j=0, \ldots, n$.

In fact,

$$
\begin{aligned}
p^{*}\left(\omega_{n}\right) & =\sum_{j=0}^{n}(-1)^{j} p_{j} d p_{0} \cdots \widehat{d p_{j}} \cdots d p_{n} \\
& =\sum_{j=0}^{n}(-1)^{j} p_{j}\left(\sum_{k=0}^{n} \frac{\partial p_{0}}{\partial X_{k}} d X_{k}\right) \cdots\left(\sum_{k=0}^{n} \widehat{\frac{\partial p_{j}}{\partial X_{k}}} d X_{k}\right) \cdots\left(\sum_{k=0}^{n} \frac{\partial p_{n}}{\partial X_{k}} d X_{k}\right) .
\end{aligned}
$$

But

$$
d X_{0} \cdots \widehat{d X_{j}} \cdots d X_{n}=(-1)^{j} X_{j} \omega_{n}
$$

and so there is a polynomial $P\left(X_{0}, \ldots, X_{n}\right)$ such that

$$
p^{*}\left(\omega_{n}\right)=P\left(X_{0}, \ldots, X_{n}\right) \omega_{n}
$$

where coefficients of $P$ belong to $\mathbb{Q}\left[a_{\alpha}^{j}\right]_{0 \leq j \leq n ; \alpha \in \mathbb{N}^{n+1}}$.

On the other hand

$$
X_{0}^{\alpha_{0}} \cdots X_{n}^{\alpha_{n}} \omega_{n} \sim 0
$$

if at least one $\alpha_{j}$ is odd.

If $\alpha_{j}=2 \beta_{j}$ with $j=0, \ldots, n$ then, in view of [8] it holds:

$$
X_{0}^{\alpha_{0}} \cdots X_{n}^{\alpha_{n}} \omega_{n} \sim \begin{cases}\frac{\alpha_{0} ! \cdots \alpha_{n} !}{\beta_{0} ! \cdots \beta_{n} !} \frac{n !}{s !} \frac{\left(\beta_{0}+\cdots+\beta_{n}+s\right) !}{\left(\alpha_{0}+\cdots+\alpha_{n}+n\right) !} \omega_{n} & \text { if } n=2 s \\ \frac{\alpha_{0} ! \cdots \alpha_{n} !}{\beta_{0} ! \cdots \beta_{n} !} \frac{s !}{2^{\alpha_{0}+\cdots+\alpha_{n}}\left(\beta_{0}+\cdots+\beta_{n}+s\right) !} \omega_{n} & \text { if } n=2 s+1\end{cases}
$$

Theorem 2.8 The Brouwer degree $B(p)$ of any polynomial map $p=\left(p_{0}, \ldots, p_{n}\right)$ : $\mathbb{S}^{n}(\mathbb{F}) \rightarrow \mathbb{S}^{n}(\mathbb{F})$ is an integer, where $\mathbb{F}$ is any field of characteristic zero.

Proof Notice that we may assume $p: \mathbb{S}^{n}\left(\mathbb{F}_{0}\right) \rightarrow \mathbb{S}^{n}\left(\mathbb{F}_{0}\right)$, where $\mathbb{F}_{0}$ is a finite extension of $\mathbb{Q}$. In particular, $\mathbb{F}_{0}$ is a subfield of some finite transcendental extension $\mathbb{F}_{0} \subseteq$ $\mathbb{C}\left(T_{1}, \ldots, T_{r}\right)$ of the complex numbers $\mathbb{C}$.

Therefore, it is enough to show that for any $p=\left(p_{0}, \ldots, p_{n}\right): \mathbb{S}^{n}\left(\mathbb{C}\left(T_{1}, \ldots, T_{r}\right)\right)$ $\rightarrow \mathbb{S}^{n}\left(\mathbb{C}\left(T_{1}, \ldots, T_{r}\right)\right)$ the Brouwer degree $B(p) \in \mathbb{Z}$.

But, there is an open subset $U \subseteq \mathbb{C}^{r}$ such that for any $t=\left(t_{1}, \ldots, t_{r}\right) \in U$ we may evaluate all coefficients of the $p_{j}$ at $t$ for $j=0, \ldots, n$ and we get $p(t): \mathbb{S}^{n}(\mathbb{C}) \rightarrow$ 
$\mathbb{S}^{n}(\mathbb{C})$, i.e., $p$ can be thought as a map $U \rightarrow \mathcal{P}_{n}(\mathbb{C})$, where $\mathcal{P}_{n}(\mathbb{C})$ is the set of polynomial maps of $\mathbb{S}^{n}(\mathbb{C})$ to itself.

Thus, $B(p)$ is now a rational function of $t$ and we have $B(p)(t)=B(p(t))$.

Finally, since $B(p): U \rightarrow \mathbb{C}$ is continuous and takes values on $\mathbb{Z}$, it is constant and so $B(p)$ is an integer.

Example 2.9 1. For a field $\mathbb{F}$, consider the ring $\mathbb{F}[i]$ with $i^{2}=-1$. Next, let $p$ : $\mathbb{S}^{n}(\mathbb{F}) \rightarrow \mathbb{S}^{n}(\mathbb{F})$ be given by

$$
p\left(x_{0}, \ldots, x_{n}\right)=\left(p_{k}\left(x_{0}\right), q_{k}\left(x_{0}\right) x_{1}, \ldots, q_{k}\left(x_{0}\right) x_{n}\right)
$$

for $\left(x_{0}, \ldots, x_{n}\right) \in \mathbb{S}^{n}(\mathbb{F})$, where $k$ is a natural number and polynomials $p_{k}, q_{k}$ are given by the formula

$$
\left(x_{0}+i x_{1}\right)^{k}=p_{k}\left(x_{0}\right)+i x_{1} q_{k}\left(x_{0}\right)
$$

for $x_{0}^{2}+x_{1}^{2}=1$. Then, we get

$$
p^{*}\left(\omega_{n}\right)=q_{k}\left(x_{0}\right)^{n-1} \omega_{n-1} \sim \begin{cases}k \omega_{n} & \text { if } n \text { is odd, } \\ \omega_{n} & \text { if } n \text { is even and } k \text { is odd } \\ 0 & \text { if } n \text { is even and } k \text { is even }\end{cases}
$$

Therefore $B(p)=k$ if $n$ is odd, $B(p)=1$ if $n$ is even and $k$ odd, and $B(p)=0$ if both $n$ and $k$ are even.

2. This example is taken from [3, p. 64]. First, notice that $i \notin \mathbb{F}$ implies $\mathbb{F}[i]=\mathbb{F}(i)$, the field extension of $\mathbb{F}$. For $\mathbb{F}$ of characteristic different from two and $n=2 m+1$, define polynomial maps $p: \mathbb{F}(i)^{2 m+2} \rightarrow \mathbb{F}(i)^{m+1}$ and $\bar{p}: \mathbb{F}(i)^{2 m+2} \rightarrow \mathbb{F}(i)^{m+1}$ by

$$
\begin{aligned}
& p\left(r_{0}, \ldots, r_{2 m+1}\right)=\left(p_{k}\left(r_{0}\right)+i q_{k}\left(r_{0}\right) r_{1}, q_{k}\left(r_{0}\right)\left(r_{2}+i r_{3}\right), \ldots, q_{k}\left(r_{0}\right)\left(r_{2 m}+i r_{2 m+1}\right)\right), \\
& \bar{p}\left(r_{0}, \ldots, r_{2 m+1}\right)=\left(p_{k}\left(r_{0}\right)+i q_{k}\left(r_{0}\right) r_{1}, q_{k}\left(r_{0}\right)\left(r_{2}-i r_{3}\right), \ldots, q_{k}\left(r_{0}\right)\left(r_{2 m}-i r_{2 m+1}\right)\right)
\end{aligned}
$$

respectively, for $\left(r_{0}, \ldots, r_{2 m+1}\right) \in \mathbb{F}^{2 m+2}$.

Next, consider a polynomial map $\tilde{p}: \mathbb{S}^{n+1}(\mathbb{F}(i)) \rightarrow \mathbb{S}^{n+1}(\mathbb{F}(i))$ given by

$$
\begin{aligned}
\tilde{p}\left(r_{0}, \ldots, r_{2 m+1}, r_{2 m+2}\right)= & \left(\frac{1}{2}\left(A_{k}\left(r_{2 m+2}\right) \bar{p}(r)+A_{k}\left(-r_{2 m+2}\right) p(r)\right),\right. \\
& \left.-\frac{i}{2}\left(A_{k}\left(-r_{2 m+2}\right) p(r)-A_{k}\left(r_{2 m+2}\right) \bar{p}(r)\right), r_{2 m+2} R_{k}\left(r_{2 m+2}^{2}\right)\right)
\end{aligned}
$$

for $\left(r_{0}, \ldots, r_{2 m+1}, r_{2 m+2}\right) \in \mathbb{S}^{n+1}(\mathbb{F}(i))$, where $r=\left(r_{0}, \ldots, r_{n}\right), A_{k} \in \mathbb{Q}[X]$ is the polynomial of algebraic degree $k-1$ given by

$$
A_{k}(X)(1+X)^{k}+A_{k}(-X)(1-X)^{k}=2
$$

and $R_{k} \in \mathbb{Q}[X]$ is given by

$$
\left(A_{k}(X)-1\right)(1+X)^{k}=X R_{k}\left(X^{2}\right)
$$


Then one gets $\tilde{p}^{*}\left(\omega_{n+1}\right) \sim k \omega_{n+1}$ and so $B(\tilde{p})=k$.

Remark 2.10 (a) $B: \mathcal{P}_{n}(\mathbb{F}) \rightarrow \mathbb{Z}$ is surjective for any field $\mathbb{F}$ and odd $n$, see [4] or [10] for explicit constructions.

(b) The image of $B: \mathcal{P}_{n}(\mathbb{F}) \rightarrow \mathbb{Z}$ contains all odd integers if $\mathbb{F}$ contains the field of real algebraic numbers $\mathbb{R}_{\text {alg }}$ and even $n$, see [4].

\section{Hopf degree of polynomial maps}

Let $q: \mathbb{S}^{2 n-1}(\mathbb{F}) \rightarrow \mathbb{S}^{n}(\mathbb{F})$ be a polynomial map. It is clear that $d\left(q^{*}\left(\omega_{n}\right)\right)=$ $q^{*}\left(d \omega_{n}\right)=0$ and then we use Theorem 2.6 to choose $\gamma_{n-1} \in \Omega^{n-1}\left(A_{2 n-1}(\mathbb{F})\right)$ such that $d \gamma_{n-1}=q^{*}\left(\omega_{n}\right)$.

Define then $H(q) \in \mathbb{F}$ by the formula

$$
\left[\gamma_{n-1} q^{*}\left(\omega_{n}\right)\right]=H(q)\left[\omega_{2 n-1}\right]
$$

By using again Theorem 2.6 it is clear the definition of $H(q)$ is correct.

If $n$ is even and we use standard de Rham cohomology, the Hopf invariant $h(g)$ of a smooth map $g: \mathbb{S}^{2 n-1} \rightarrow \mathbb{S}^{n}$ (see e.g., [6, p. 229]) is given by

$$
\left[\frac{\gamma_{n-1}}{\operatorname{vol}\left(\mathbb{S}^{n}\right)} g^{*}\left(\frac{\omega_{n}}{\operatorname{vol}\left(\mathbb{S}^{n}\right)}\right)\right]=h(g)\left[\frac{\omega_{2 n-1}}{\operatorname{vol}\left(\mathbb{S}^{2 n-1}\right)}\right]
$$

because we have to replace the volume form $\omega_{n}$ by the orientation form $\alpha_{n}=\frac{\omega_{n}}{\operatorname{vol}\left(\mathbb{S}^{n}\right)}$ whose integral over the sphere is one. Therefore,

$$
H(g)=\frac{\operatorname{vol}^{2}\left(\mathbb{S}^{n}\right)}{\operatorname{vol}\left(\mathbb{S}^{2 n-1}\right)} h(g)=\frac{4^{n}}{m\left(\begin{array}{l}
n \\
m
\end{array}\right)} h(g)
$$

for $n=2 m$.

We use now the previous formula for defining the Hopf invariant $h(q)$ of any polynomial map $q: \mathbb{S}^{2 n-1}(\mathbb{F}) \rightarrow \mathbb{S}^{n}(\mathbb{F})$ for $n$ even. This could be done because $\frac{\operatorname{vol}^{2}\left(\mathbb{S}^{n}\right)}{\operatorname{vol}\left(\mathbb{S}^{2 n-1}\right)}$ is then a rational number.

Of course, we know that for $\mathbb{F}$ being the real or complex field, $H(q)=0$ for odd $n$ and the Hopf invariant $h(q)$ is an integer for even $n$. Then, by using the same method of proof as that of Theorem 2.8, we have the following:

Theorem 3.1 For a field $\mathbb{F}$ of characteristic zero and any even $n$, the Hopf degree $h(q)$ of any polynomial map $q: \mathbb{S}^{2 n-1}(\mathbb{F}) \rightarrow \mathbb{S}^{n}(\mathbb{F})$ is an integer.

Example 3.2 We mimic the Hopf maps $\mathbb{S}^{3} \longrightarrow \mathbb{S}^{2}, \mathbb{S}^{7} \longrightarrow \mathbb{S}^{4}$ and $\mathbb{S}^{15} \longrightarrow \mathbb{S}^{8}$ to analyse the following polynomial maps.

1. Let $q: \mathbb{S}^{3}(\mathbb{F}) \rightarrow \mathbb{S}^{2}(\mathbb{F})$ be given by

$$
q\left(x_{0}, x_{1}, x_{2}, x_{3}\right)=\left(2\left(x_{0} x_{2}+x_{1} x_{3}\right), 2\left(-x_{0} x_{3}+x_{1} x_{2}\right), x_{0}^{2}+x_{1}^{2}-x_{2}^{2}-x_{3}^{2}\right) .
$$


A straightforward calculation gives

$$
q^{*}\left(\omega_{2}\right)=-4\left(d X_{0} d X_{1}+d X_{2} d X_{3}\right)=d \gamma_{1}
$$

with $\gamma_{1}=-4\left(X_{0} d X_{1}+X_{2} d X_{3}\right)$.

But,

$$
\begin{aligned}
& d X_{1} d X_{2} d X_{3}=X_{0} \omega_{3}, \quad d X_{0} d X_{2} d X_{3}=-X_{1} \omega_{3}, \quad d X_{0} d X_{1} d X_{3}=X_{2} \omega_{3}, \\
& d X_{0} d X_{1} d X_{2}=-X_{3} \omega_{3}
\end{aligned}
$$

and

$$
X_{0}^{2} \omega_{3} \sim X_{1}^{2} \omega_{3} \sim X_{2}^{2} \omega_{3} \sim X_{3}^{2} \omega_{3}
$$

Therefore, we have

$$
\gamma_{1} q^{*}\left(\omega_{2}\right)=16\left(X_{0}^{2}+X_{2}^{2}\right) \omega_{3} \sim 8\left(X_{0}^{2}+X_{1}^{2}+X_{2}^{2}+X_{3}^{2}\right) \omega_{3}=8 \omega_{3} .
$$

Thus $H(q)=8$ and so

$$
h(q)=1
$$

as expected.

2. Consider the non-commutative and unitary ring $\mathbb{F}\{i, j, k\}$ with $i^{2}=j^{2}=k^{2}=$ $-1, i j=k, j k=i, k i=j$. Given $s=x_{0}+i x_{1}+j x_{2}+k x_{3} \in \mathbb{F}\{i, j, k\}$, we write $|s|^{2}=x_{0}^{2}+x_{1}^{2}+x_{2}^{2}+x_{3}^{2}$ and $\bar{s}=x_{0}-i x_{1}-j x_{2}-k x_{3}$. Then, $s \bar{s}=|s|^{2}$, $\left|s_{1} s_{2}\right|^{2}=\left|s_{1}\right|^{2}\left|s_{2}\right|^{2}$ for $s, s_{1}, s_{2} \in \mathbb{F}\{i, j, k\}$ and

$$
\mathbb{S}^{7}(\mathbb{F}) \cong\left\{\left(s_{0}, s_{1}\right) \in \mathbb{F}\{i, j, k\} \times \mathbb{F}\{i, j, k\} ;\left|s_{0}\right|^{2}+\left|s_{1}\right|^{2}=1\right\} .
$$

Now, let $q: \mathbb{S}^{7}(\mathbb{F}) \rightarrow \mathbb{S}^{4}(\mathbb{F})$ be given by

$$
q\left(x_{0}, x_{1}, x_{2}, x_{3}, x_{4}, x_{5}, x_{6}, x_{7}\right)=\left(2 s_{0} \bar{s}_{1},\left|s_{0}\right|^{2}-\left|s_{1}\right|^{2}\right)
$$

for $\left(x_{0}, x_{1}, x_{2}, x_{3}, x_{4}, x_{5}, x_{6}, x_{7}\right) \in \mathbb{S}^{7}(\mathbb{F})$, where $s_{0}=x_{0}+i x_{1}+j x_{2}+k x_{3}$ and $s_{1}=x_{4}+i x_{5}+j x_{6}+k x_{7}$.

Then, we get $H(q)=\frac{64}{3}$ and so $h(q)=1$.

3. Next, consider the non-associative and unitary ring $\mathbb{F}\left\{e_{1}, \ldots, e_{7}\right\}$, where products $e_{s} e_{t}$ are defined by the Cayley algebra rules for $s, t=1, \ldots, 7$. Given $c=x_{0}+$ $e_{1} x_{1}+\cdots+e_{7} x_{7} \in \mathbb{F}\left\{e_{1}, \ldots, e_{7}\right\}$, write $|c|^{2}=x_{0}^{2}+x_{1}^{2}+\cdots+x_{7}^{2}$ and $\bar{c}=$ $x_{0}-e_{1} x_{1}-\cdots-e_{7} x_{7}$. Then, $c \bar{c}=|c|^{2},\left|c_{1} c_{2}\right|^{2}=\left|c_{1}\right|^{2}\left|c_{2}\right|^{2}$ for $c_{1}, c_{2} \in \mathbb{F}\left\{e_{1}, \ldots, e_{7}\right\}$ and

$$
\mathbb{S}^{15}(\mathbb{F}) \cong\left\{\left(c_{0}, c_{1}\right) \in \mathbb{F}\left\{e_{1}, \ldots, e_{7}\right\} \times \mathbb{F}\left\{e_{1}, \ldots, e_{7}\right\} ;\left|c_{0}\right|^{2}+\left|c_{1}\right|^{2}=1\right\} .
$$


Now, let $q: \mathbb{S}^{15}(\mathbb{F}) \rightarrow \mathbb{S}^{8}(\mathbb{F})$ be given by

$$
q\left(x_{0}, \ldots, x_{15}\right)=\left(2 c_{0} \bar{c}_{1},\left|c_{0}\right|^{2}-\left|c_{1}\right|^{2}\right)
$$

for $\left(x_{0}, \ldots, x_{15}\right) \in \mathbb{S}^{15}(\mathbb{F})$, where $c_{0}=x_{0}+e_{1} x_{1}+\cdots+e_{7} x_{7}$ and $c_{1}=x_{8}+e_{1} x_{9}+$ $\cdots+e_{7} x_{15}$.

Then, we get $H(q)=\frac{4^{7}}{70}$ and so $h(q)=1$.

We close the paper with:

Remark 3.3 Observe that proofs of Theorems 2.8 and 3.1 use topology. It would be interesting having algebraic ones.

Acknowledgments The authors are indebted to the referee for his/her many detailed suggestions which lead to a considerable improvement of the original version of the paper.

Open Access This article is distributed under the terms of the Creative Commons Attribution License which permits any use, distribution, and reproduction in any medium, provided the original author(s) and the source are credited.

\section{References}

1. Bourbaki, N.: Algèbre. Chapitre X, Algèbre homologique. Masson, Paris (1980)

2. Bredon, G.E.: Introduction to compact transformation groups. Academic Press, New York (1972)

3. Golasiński, M., Gómez Ruiz, F.: Polynomial and regular maps into Grassmannians. K-Theory 26(1), 51-68 (2002)

4. Golasiński, M., Gómez Ruiz, F.: On the algebraic K-theory of $R[X, Y, Z] /\left(X^{2}+Y^{2}+Z^{2}-1\right)$. Bull. Belg. Math. Soc. Simon Stevin 18, 849-860 (2011)

5. Gómez Ruiz, F.: The number of generators of the algebra of Kähler differentials. Demonstr. Math. XXIII(2), 375-383 (1990)

6. Greub, W., Halperin, S., Vanstone, R.: Connections, curvature and cohomology. vol. 1, De Rham cohomology of manifolds and vector bundles. Academic Press, New York (1972)

7. Hirsch, M.W.: Differential Topology. Springer, New York (1976)

8. Kong, M.: Euler classes of inner product modules. J. Algebra 49(1), 276-303 (1977)

9. Wood, R.: Polynomial maps from spheres to spheres. Invent. Math. 5, 163-168 (1968)

10. Wood, R.: Polynomial maps of affine quadrics. Bull. Lond. Math. Soc. 25(5), 491-497 (1993) 